IJMMS 30:11 (2002) 651-657

PII. S0161171202011523

http://ijmms.hindawi.com

(c) Hindawi Publishing Corp.

\title{
ON GENERALIZED FUZZY STRONGLY SEMICLOSED SETS IN FUZZY TOPOLOGICAL SPACES
}

\author{
OYA BEDRE OZBAKIR
}

Received 22 January 2001 and in revised form 2 October 2001

\begin{abstract}
We introduce the concept of generalized fuzzy strongly semiclosed, generalized fuzzy almost-strongly semiclosed, generalized fuzzy strongly closed, and generalized fuzzy almost-strongly closed sets. In the light of these definitions, we also define some generalizations of fuzzy continuous functions and discuss the relations between these new classes of functions and other fuzzy continuous functions.
\end{abstract}

2000 Mathematics Subject Classification: 54A40, 03E72.

1. Introduction and preliminaries. Generalized semiclosed (semiopen) and generalized closed (open) sets play an important role in general topology [2, 9]. Balasubramanian and Sundaram [6] defined generalized fuzzy closed set in fuzzy topological spaces. Later, Abd El-Hakeim [1] introduced the generalized fuzzy semiclosed, generalized fuzzy weakly semiclosed, and generalized fuzzy regular closed sets and studied some of their properties.

In Section 2, we introduce generalized fuzzy strongly semiclosed, generalized fuzzy almost-strongly semiclosed, generalized fuzzy strongly closed, and generalized fuzzy almost-strongly closed sets and establish some of their properties. (We have not seen such discussions on the properties of these sets in general topological spaces.) We also discuss the relations between fuzzy closed sets [3], fuzzy semiclosed sets [3], and fuzzy strongly semiclosed sets [4].

In Section 3, we introduce four new classes of functions among fuzzy topological spaces which are weaker than the classes of fuzzy continuous functions, fuzzy strongly semicontinuous, and fuzzy semicontinuous functions, respectively. (We have not seen corresponding concepts in general topological spaces.) Also, some examples are given, and relationships between these new classes and other classes of fuzzy continuous functions are obtained.

For $X, I^{X}$ denotes the collection of all mappings from $X$ into $I=[0,1]$. A member $\lambda$ of $I^{X}$ is called a fuzzy set of $X$. By $(X, \tau)$ or simply by $X$, we denote a fuzzy topological space (FTS) due to Chang [7]. The interior, the closure, and the complement of a fuzzy set $\mu \in I^{X}$ will be denoted by int $\mu, \operatorname{cl} \mu$, and $\mu^{\prime}$, respectively.

Now we introduce some basic notions and results that are used in the sequel.

DEFINITION 1.1. A fuzzy set $\mu$ of an FTS $(X, \tau)$ is said to be

(a) fuzzy semiopen if and only if $\mu \leq \operatorname{cl}(\operatorname{int} \mu)$ [3];

(b) fuzzy strongly semiopen if and only if there is a $\beta \in \tau$ such that $\beta \leq \mu \leq$ $\operatorname{int}(\operatorname{cl} \beta)[4]$; 
(c) fuzzy strongly semiclosed if and only if there is a fuzzy closed set $\beta \in I^{X}$ such that $\operatorname{cl}(\operatorname{int} \beta) \leq \mu \leq \beta[4]$.

Definition 1.2. A fuzzy set $\lambda$ of an FTS $(X, \tau)$ is said to be

(a) generalized fuzzy closed (gf-closed) if and only if $\mathrm{cl} \lambda \leq \beta$ whenever $\lambda \leq \mu$ and $\mu$ is fuzzy open [6];

(b) generalized fuzzy semiclosed (gf-semiclosed) if and only if $\operatorname{cl} \lambda \leq \mu$ whenever $\lambda \leq \mu$ and $\mu$ is a fuzzy semiopen set [1].

The family of all fuzzy semiopen, fuzzy semiclosed, fuzzy strongly semiopen, and fuzzy strongly semiclosed sets of an $\operatorname{FTS}(X, \tau)$ will be denoted by $\operatorname{FSO}(X), \operatorname{FSC}(X)$, $\operatorname{FSTSO}(X)$, and $\operatorname{FSTSC}(X)$, respectively.

Definition 1.3 (see [10]). Let $\mu$ be a fuzzy set in an FTS $(X, \tau)$. Then

$$
\operatorname{sint} \mu=\vee\{\beta: \beta \leq \mu, \beta \text { is fuzzy semiopen }\}
$$

is called a fuzzy semi-interior of $\mu$,

$$
\operatorname{scl} \mu=\wedge\{\beta: \mu \leq \beta, \beta \text { is fuzzy semiclosed }\}
$$

is called a fuzzy semiclosure of $\mu$.

LEMMA 1.4 (see [5]). For a fuzzy set $\mu$ of an $\operatorname{FTS}(X, \tau)$,

(a) $(\operatorname{sint} \mu)^{\prime}=\operatorname{scl}\left(\mu^{\prime}\right)$,

(b) $(\operatorname{scl} \mu)^{\prime}=\operatorname{sint}\left(\mu^{\prime}\right)$.

Definition 1.5 (see [8]). Let $\mu$ be a fuzzy set in an FTS $(X, \delta)$ and define the following fuzzy subsets:

$$
\text { stsint } \mu=\vee\{\beta: \beta \leq \mu, \beta \text { is fuzzy strongly semiopen }\}
$$

is called the fuzzy strong semi-interior of $\mu$,

$$
\operatorname{stscl} \mu=\wedge\left\{\beta: \mu \leq \beta, \beta^{\prime} \text { is fuzzy strongly semiopen }\right\}
$$

is called the fuzzy strong semiclosure of $\mu$.

Proposition 1.6 (see [8]). Let $\mu$ and $\beta$ be fuzzy sets in an FTS $(X, \tau)$. Then the following statements are valid:

(a) int $\mu \leq \operatorname{stsint} \mu \leq \operatorname{sint} \mu \leq \mu \leq \operatorname{scl} \mu \leq \operatorname{stscl} \mu \leq \operatorname{cl} \mu$;

(b) $\mu \leq \beta \Rightarrow \operatorname{stsint} \mu \leq \operatorname{stsint} \beta$; $\operatorname{stscl} \mu \leq \operatorname{stscl} \beta$;

(c) $\operatorname{st\operatorname {sin}t} 0=0$; $\operatorname{stscl} 0=0$; $\operatorname{stsint} 1=1$; $\operatorname{stscl} 1=1$;

(d) $\operatorname{stsint}(\operatorname{stsin} t \mu)=\operatorname{stsint} \mu ; \operatorname{stscl}(\operatorname{stscl} \mu)=\operatorname{stscl} \mu$;

(e) $\mu$ is fuzzy strongly semiopen if and only if $\operatorname{stsint} \mu=\mu$;

(f) $\mu$ is fuzzy strongly semiclosed if and only if $\operatorname{stscl} \mu=\mu$;

(g) $\operatorname{stsint}(\mu \wedge \beta) \leq \operatorname{stsint} \mu \wedge \operatorname{stsint} \beta$; $\operatorname{stscl}(\mu \vee \beta) \geq \operatorname{stscl} \mu \vee \operatorname{stscl} \beta$.

DEFINITION 1.7. Let $f:(X, \tau) \rightarrow(Y, \delta)$ be a mapping from an FTS $(X, \tau)$ to another FTS $(Y, \delta)$. Then $f$ is called

(a) a fuzzy continuous mapping if $f^{-1}(\mu) \in \tau$ for each $\mu \in \delta$ [7]; 
(b) a fuzzy semicontinuous mapping if $f^{-1}(\mu) \in \operatorname{FSO}(X)$ for each $\mu \in \delta$ [7];

(c) a fuzzy strongly semicontinuous mapping if $f^{-1}(\mu) \in \operatorname{FSTSO}(X)$ for each $\mu \in$ $\delta$ [3].

REMARK 1.8. Every fuzzy open (closed) set is a fuzzy strongly semiopen (semiclosed) set. Every fuzzy strongly semiopen (semiclosed) set is a fuzzy semiopen (semiclosed) set [8].

\section{Generalized fuzzy strongly semiclosed sets in fuzzy topological spaces}

DEFINITION 2.1. A fuzzy subset $\mu$ of an FTS topological space $(X, \tau)$ is called

(a) generalized fuzzy strongly semiclosed (gfst-semiclosed) if and only if $\operatorname{cl}(\mu) \leq \lambda$ whenever $\mu \leq \lambda$ and $\lambda \in \operatorname{FSTSO}(X)$;

(b) generalized fuzzy almost-strongly semiclosed (gfast-semiclosed) if and only if $\operatorname{stscl}(\mu) \leq \lambda$ whenever $\mu \leq \lambda$ and $\lambda \in \operatorname{FSTSO}(X)$;

(c) a generalized fuzzy strongly closed (gfst-closed) if and only if $\operatorname{stscl}(\mu) \leq \lambda$ whenever $\mu \leq \lambda$ and $\lambda \in \tau$;

(d) a generalized fuzzy almost-strongly closed (gfast-closed) if and only if $\operatorname{scl} \mu \leq \lambda$ whenever $\mu \leq \lambda$, and $\lambda \in \operatorname{FSTSO}(X)$.

It is clear from Remark 1.8 and Definition 2.1 that the following diagram implications are true:

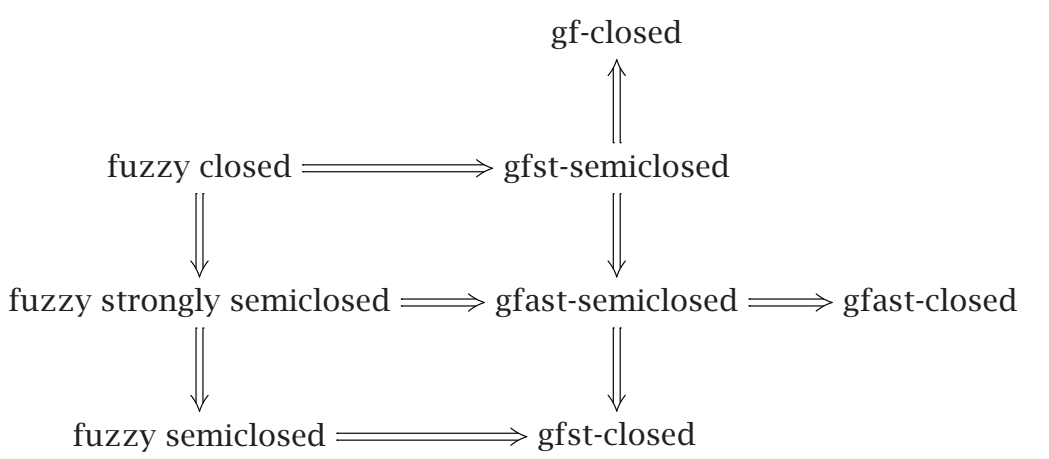

The following examples show that the reverse may not be true in general.

EXAMPLE 2.2. Consider the set $X=\left\{x_{1}, x_{2}, x_{3}\right\}$. Define $\lambda, \mu \in I^{X}$ as follows: $\mu\left(x_{2}\right)=$ $\mu\left(x_{3}\right)=0, \mu\left(x_{1}\right)=1 ; \lambda\left(x_{1}\right)=\lambda\left(x_{2}\right)=1, \lambda\left(x_{3}\right)=0$, and $\tau=\{0,1, \mu\}$. It is easy to see that $\lambda$ is a gf-closed set but it is neither gfsts-closed nor fuzzy closed.

EXAMPLE 2.3. Let $X=\{a, b, c\}$, and let $\lambda, \mu \in I^{X}$ be defined as follows:

$$
\begin{array}{lll}
\mu(a)=0.4, & \mu(b)=0.2, & \mu(c)=0.2 ; \\
\lambda(a)=0.5, & \lambda(b)=0.4, & \lambda(c)=0.6 .
\end{array}
$$

We define $\tau=\{0,1, \lambda\}$. Since $\lambda$ is only a fuzzy open set, we have $\operatorname{FSTSO}(X)=\{\alpha$ : $\alpha(a) \in[0.5,1], \alpha(b) \in[0.4,1], \alpha(c) \in[0.6,1]\}$. Hence for every $\alpha \in \operatorname{FSTO}(X), \mu \leq \alpha$ implies that $\mu$ is gfast-semiclosed, but it is not gfst-semiclosed. Because $\operatorname{cl}(\mu)=\lambda^{\prime}$ for some $\alpha_{1} \in \operatorname{FSTSO}(X)$, we have $\lambda^{\prime} \nless \alpha_{1}$ where $\alpha_{1}(a)=0.5, \alpha_{1}(b)=0.4, \alpha_{1}(c)=0.8$. 
EXAMPLE 2.4. Let $X=\{a, b\}$. Define $\tau=\{0,1, \lambda\}$ where $\lambda \in I^{X}$ such that $\lambda(a)=0.3$, $\lambda(b)=0.6$, and define $\beta \in I^{X}$ such that $\beta(a)=\beta(b)=0.5$. It is easy to check that $\beta$ is not a gfast-semiclosed set. However, $\beta$ is gfst-closed since the only fuzzy open set containing $\lambda$ is 1 itself.

EXAMPLE 2.5. Let $X=\{a, b\}$. Define $\tau=\{0,1, \beta\}$ where $\beta \in I^{X}$ such that $\beta(a)=0.3$, $\beta(b)=0.5 ; \lambda \in I^{X}$ such that $\lambda(a)=0.2, \lambda(b)=0.5$.

It is easy to see that $\lambda$ is gfast-closed but not gfast-semiclosed.

EXAMPLE 2.6. Let $\mu$ and $\gamma$ be two fuzzy subsets of $I=[0,1]$ defined as follows: for each $x \in I$

$$
\mu(x)= \begin{cases}1-2 x, & 0 \leq x \leq \frac{1}{2}, \\ 0, & \frac{1}{2} \leq x \leq 1,\end{cases}
$$

where $\gamma(x)=1-x$. We consider the fuzzy topology $\tau=\{0,1, \gamma\}$. It is clear that $\mu$ is gfast-semiclosed since $\operatorname{stscl} \mu=\gamma \leq \alpha$ whenever $\mu \leq \alpha$ and $\alpha \in \operatorname{FSTSO}(X)$, where $\operatorname{FSTSO}(X)=\{\alpha: \gamma \leq \alpha \leq 1\}$. But $\mu$ is not fst-semiclosed.

EXAMPLE 2.7. Let $X=\{a, b, c\}$. Define $\tau=\{0,1, \mu\}$ where $\mu(a)=0.3, \mu(b)=0.5$, $\mu(c)=0.2$, and $\lambda(a)=0.5, \lambda(b)=0.3, \lambda(c)=0.2$. Then $\lambda$ is a gfst-closed set but not a fuzzy semiclosed set.

THEOREM 2.8. The union of two gfst-semiclosed sets is a gfst-semiclosed set.

Proof. The proof is straightforward.

However, the intersection of two gfst-semiclosed sets is not a gfst-semiclosed set. We can see this in the following example.

EXAMPLE 2.9. Let $X=\{a, b\}$, and let $\lambda, \beta_{1}, \beta_{2} \in I^{X}$ be defined as follows:

$$
\begin{aligned}
\lambda(a) & =0.5, & \lambda(b)=0 ; \\
\beta_{1}(a)=0.2, & & \beta_{1}(b)=1 ; \\
\beta_{2}(a)=0.7, & & \beta_{2}(b)=0 .
\end{aligned}
$$

Consider the fuzzy topology $\tau=\{0,1, \lambda\}$. It is clear that $\beta_{1}, \beta_{2}$ are gfst-semiclosed sets but $\beta_{1} \wedge \beta_{2}$ is not.

THEOREM 2.10. Let $(X, \tau)$ be an FTS and $\mu \in I^{X}$. If $\mu$ is gfst-semiclosed, and $\mu \leq \lambda \leq$ $\mathrm{cl} \mu$, then $\lambda$ is gfst-semiclosed.

Proof. Let $\beta \in \operatorname{FSTSO}(X)$ such that $\lambda \leq \beta$. We must show that $\mathrm{cl} \lambda \leq \beta$. Since $\mu \leq \lambda$, $\mu \leq \beta$, and $\mu$ is a gfst-semiclosed set $\operatorname{cl} \mu \leq \beta$. But $\operatorname{cl} \lambda \leq \operatorname{cl} \mu$ and $\lambda \leq \operatorname{cl} \mu, \operatorname{cl} \lambda \leq \beta$, therefore $\lambda$ is gfst-semiclosed.

REMARK 2.11. The complement of a gfst-semiclosed set (resp., gfast-semiclosed, gfst-closed, and gfast-closed) is a gfst-semiopen one (resp., gfast-semiopen, gfst-open, and gfast-open) set. 
THEOREM 2.12. A fuzzy set $\mu \in I^{X}$ is gfst-semiopen if and only if $\beta \leq \operatorname{int} \mu$ whenever $\beta \in \operatorname{FSTSC}(X)$, and $\beta \leq \mu$.

Proof. Let $\mu$ be gfst-semiopen and $\beta$ fuzzy strongly semiclosed such that $\beta \leq \mu$ implies that $1-\beta \geq 1-\mu$, and $1-\mu$ is a gfst-semiclosed set. Hence we have $\operatorname{cl}(1-\mu) \leq$ $1-\beta, 1-\operatorname{cl}(1-\mu) \geq 1-(1-\beta)=\beta$. We know that $1-\operatorname{cl}(1-\mu)=\operatorname{int} \mu$. Thus $\beta \leq \operatorname{int} \mu$.

Conversely, suppose that $\beta \leq \operatorname{int} \mu$ whenever $\beta$ is a fuzzy strongly semiclosed set and $\beta \leq \mu$. We must show that $1-\mu$ is a gfst-semiclosed set. Let $1-\mu \leq \alpha$ whenever $\alpha$ is fuzzy strongly semiopen. Since $1-\mu \leq \alpha$ implies that $1-\alpha \leq \mu$. By the hypothesis we have $1-\alpha \leq \mu$ or $1-\operatorname{int} \mu \leq \alpha$. Hence $1-\operatorname{int} \mu=\operatorname{cl}(1-\mu)$, which implies that $\operatorname{cl}(1-\mu) \leq \alpha$. Thus $1-\mu$ is gfst-semiclosed.

TheOrem 2.13. Let $(X, \tau)$ be a fuzzy topological space. A fuzzy set $\mu \in I^{X}$ is a gfastsemiopen (resp., gfst-open, gfast-open) set if and only if $\beta \leq \operatorname{stsint} \mu$ (resp., $\beta \leq \operatorname{stsint} \mu$, $\beta \leq \operatorname{sint} \mu$ ) whenever $\beta \in \operatorname{FSTSC}(X)$ (resp., $\beta \in \mathrm{FC}(X), \beta \in \operatorname{FSTSC}(X))$ set and $\beta \leq \mu$.

Proof. The proof is similar to the proof of Theorem 2.12 .

3. Generalized fuzzy strongly semicontinuous functions. In this section, four new classes of functions are introduced. Their relationships with other fuzzy continuous functions are established.

DeFinITION 3.1. Let $(X, \tau)$ and $(Y, \phi)$ be two fuzzy topological spaces. A mapping $f:(X, \tau) \rightarrow(Y, \phi)$ is called

(a) generalized fuzzy strongly semicontinuous (gfst-semicontinuous) if the inverse image of every fuzzy closed set in $Y$ is a gfst-semiclosed set in $X$;

(b) generalized fuzzy almost-strongly semicontinuous (gfast-semicontinuous) if the inverse image of every fuzzy closed set in $Y$ is a gfast-semiclosed set in $X$;

(c) generalized fuzzy strongly-continuous (gfst-continuous) if the inverse image of every fuzzy closed set in $Y$ is a gfst-closed set in $X$;

(d) generalized fuzzy almost-strongly continuous (gfast-continuous) if the inverse image of every fuzzy closed in $Y$ is a gfast-closed set in $X$.

Thus we have the following diagram:

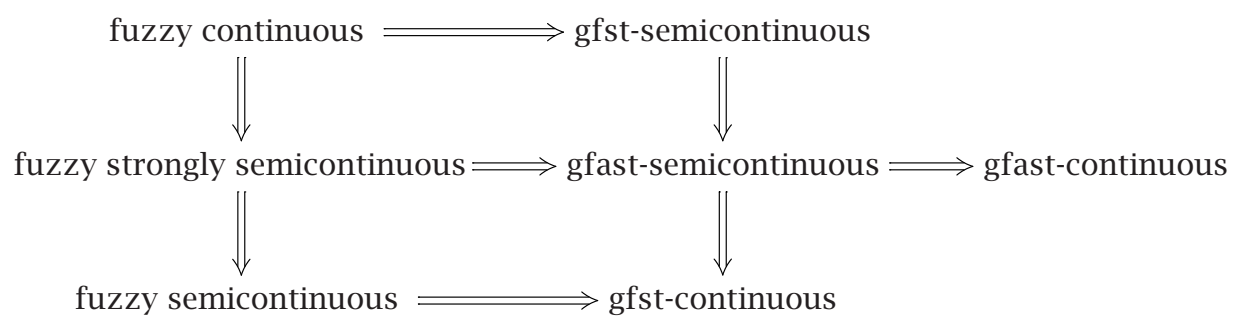

None of these implications are reversible, as the following counterexamples state.

EXAMPLE 3.2. Let $X=\{a, b, c\}, Y=\{p, q\}$. Define $\tau=\{0,1, \lambda\}$, and $\phi=\{0,1, \mu\}$ are FTS on $X$ and $Y$, respectively, where $\lambda \in I^{X}$ is such that $\lambda(a)=0, \lambda(b)=\lambda(c)=0.5$, 
and $\mu \in I^{Y}$ is such that $\mu(p)=0, \mu(q)=1$. A mapping $f: X \rightarrow Y$ is defined as $f(a)=$ $f(b)=p, f(c)=q$.

It is clear that $f$ is gfst-semicontinuous but not fuzzy continuous.

EXAMPLE 3.3. Let $X=\{a, b\}$. Define $\tau=\{0,1, \beta\}, \phi=\{0,1, v\}$ where $\beta, v \in I^{X}$ are such that $\beta(a)=0.5, \beta(b)=0.3 ; v(a)=0.7, v(b)=0.6$. A mapping $f: X \rightarrow X$ is defined as $f(a)=b, f(b)=a$. So $f$ is gfst-continuous but not fuzzy semicontinuous.

EXAMPLE 3.4. Let $X=\{a, b\}, Y=\{p, q\}$. Define $\tau=\{0,1, \mu\}, \phi=\{0,1, \nu\}$ such that $\mu(a)=0.3, \mu(b)=0.6 ; \nu(p)=0.4, v(q)=0.6$. Also define $f: X \rightarrow Y$ as $f(a)=p$, $f(b)=q$. Then $f$ is gfast-semicontinuous. But it is not gfst-semicontinuous since $\operatorname{cl} f^{-1}\left(\nu^{\prime}\right) \nless \alpha$ whenever $f^{-1}\left(\nu^{\prime}\right) \leq \alpha$, and $\alpha \in \operatorname{FSTSO}(X)$ such that $\alpha(a)=0.6, \alpha(b)=$ 0.4 .

EXAMPLE 3.5. Let $X=\{a, b\}, Y=\{x, y\}$. The fuzzy sets $\mu \in I^{X}$ and $\gamma \in I^{Y}$ are defined as follows:

$$
\begin{array}{ll}
\mu(a)=0.3, & \mu(b)=0.7 ; \\
\gamma(x)=0.2, & \gamma(y)=0.5 .
\end{array}
$$

Consider the fuzzy topologies $\tau=\{0,1, \mu\}$ and $\phi=\{0,1, \gamma\}$. The mapping $f: X \rightarrow Y$ is defined as $f(a)=x, f(b)=y$. It is clear that $f$ is gfst-continuous but not gfastsemicontinuous since stscl $f^{-1}\left(\gamma^{\prime}\right)=1 \nless \alpha$ where $f^{-1}\left(\gamma^{\prime}\right) \leq \alpha$ for $\alpha \in \operatorname{FSTSO}(X)$ such that $\alpha(a)=0.8, \alpha(b)=0.7$.

EXAMPLE 3.6. Let the sets $X$ and $Y$ be the same as in Example 3.2. The fuzzy sets $\mu \in I^{X}$ and $\beta \in I^{Y}$ are defined as follows:

$$
\begin{array}{ll}
\mu(a)=0.5, \quad \mu(b)=0.4, \quad \mu(c)=0 ; \\
\beta(p)=0.7, \quad \beta(q)=0.9 .
\end{array}
$$

If we define $f: X \rightarrow Y$ satisfying $f(a)=f(c)=p, f(b)=q$, then $f$ is fuzzy gfastsemicontinuous but not fuzzy strongly semicontinuous since $f^{-1}\left(\beta^{\prime}\right) \notin \operatorname{FSTSC}(X)$.

EXAMPLE 3.7. Consider the same sets of Example 3.4. Let $\tau=\{0,1, \mu\}, \phi=\{0,1, \beta\}$ where $\mu(a)=0.6, \mu(b)=0.3 ; \beta(p)=0.7, \beta(q)=0$. Consider the mapping $f: X \rightarrow Y$ defined as $f(a)=p, f(b)=q$. It is clear that $f$ is gfast-continuous but not gfastsemicontinuous.

Theorem 3.8. Let $f: X \rightarrow Y$ and $g: Y \rightarrow Z$ be mappings, where $X, Y$, and $Z$ are FTS's. If $f$ is gfst-semicontinuous and $g$ is fuzzy continuous then $g \circ f$ is gfstsemicontinuous.

Proof. It is easy since we have,

$$
(g \circ f)^{-1}(\mu)=f^{-1}\left(g^{-1}(\mu)\right) \text { for each fuzzy closed set } \mu \text { of } Z \text {. }
$$

Theorem 3.8 is not valid if $g$ is gfst-semicontinuous. 
EXAMPLE 3.9. Let $X=\{a \cdot b \cdot c\}$. Define $\tau_{1}=\{0,1, \lambda\}$ where $\lambda \in I^{X}$ is such that $\lambda(a)=\lambda(b)=1$, and $\lambda(c)=0$. Let $X=\{a, b, c\}$ and $\lambda, \mu, \gamma$, and $\beta$ be fuzzy sets defined as follows: $\lambda(a)=\lambda(b)=1, \lambda(c)=0 ; \mu(a)=0, \mu(b)=\mu(c)=1 ; \gamma(a)=1$, $\gamma(b)=\gamma(c)=0$ and $\beta(a)=\beta(b)=1, \beta(c)=0$. Let $\tau_{1}=\{0,1, \lambda\}, \tau_{2}=\{0,1, \mu, \gamma\}$, and $\tau_{3}=\{0,1, \beta\}$.

Also define $f:\left(X, \tau_{1}\right) \rightarrow\left(X, \tau_{2}\right)$ as $f(a)=f(c)=c, f(b)=b$ and let $g:\left(X, \tau_{2}\right) \rightarrow$ $\left(X, \tau_{3}\right)$ be the identity map. Then $f$ and $g$ are gfst-semicontinuous but $g \circ f$ is not gfst-semicontinuous since $\beta^{\prime}$ is fuzzy closed in $\left(X, \tau_{3}\right),(g \circ f)^{-1}\left(\beta^{\prime}\right)=\beta^{\prime}$ is not gfstsemiclosed in $\left(X, \tau_{1}\right)$.

ACKNOWLEDGment. The author would like to thank the referees for the helpful suggestions.

\section{REFERENCES}

[1] K. M. Abd El-Hakeim, Generalized semi-continuous mappings in fuzzy topological spaces, J. Fuzzy Math. 7 (1999), no. 3, 577-589.

[2] S. P. Arya and T. M. Nour, Characterizations of s-normal spaces, Indian J. Pure Appl. Math. 21 (1990), no. 8, 717-719.

[3] K. K. Azad, On fuzzy semicontinuity, fuzzy almost continuity and fuzzy weakly continuity, J. Math. Anal. Appl. 82 (1981), no. 1, 14-32.

[4] S. Z. Bai, Fuzzy strongly semiopen sets and fuzzy strong semicontinuity, Fuzzy Sets and Systems 52 (1992), no. 3, 345-351.

[5] _ _ Fuzzy weak semicontinuity, Fuzzy Sets and Systems 47 (1992), no. 1, 93-98.

[6] G. Balasubramanian and P. Sundaram, On some generalizations of fuzzy continuous functions, Fuzzy Sets and Systems 86 (1997), no. 1, 93-100.

[7] C. L. Chang, Fuzzy topological spaces, J. Math. Anal. Appl. 24 (1968), 182-190.

[8] B. Krsteska, Fuzzy strongly preopen sets and fuzzy strong precontinuity, Mat. Vesnik 50 (1998), no. 3-4, 111-123.

[9] N. Levine, Generalized closed sets in topology, Rend. Circ. Mat. Palermo (2) 19 (1970), 89-96.

[10] T. H. Yalvaç, Semi-interior and semiclosure of a fuzzy set, J. Math. Anal. Appl. 132 (1988), no. 2, 356-364.

Oya Bedre Ozbakir: Department of Mathematics, Ege University, 35100 BornovaIZMIR, TURKEY

E-mail address: ozbakir@sci.ege.edu.tr 


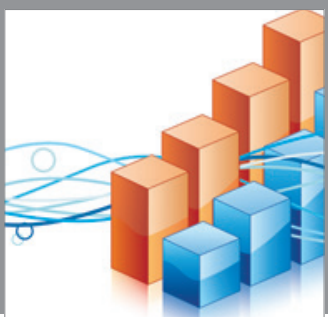

Advances in

Operations Research

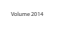

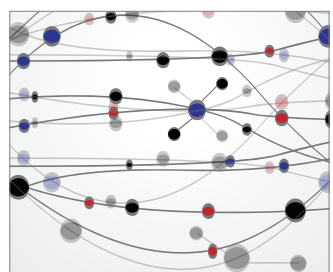

\section{The Scientific} World Journal
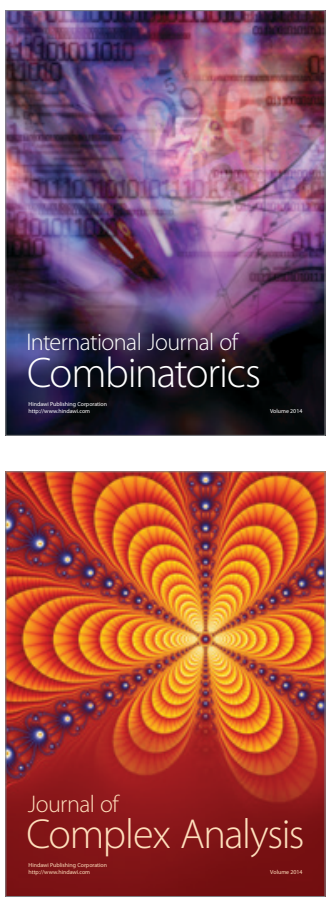

International Journal of

Mathematics and

Mathematical

Sciences
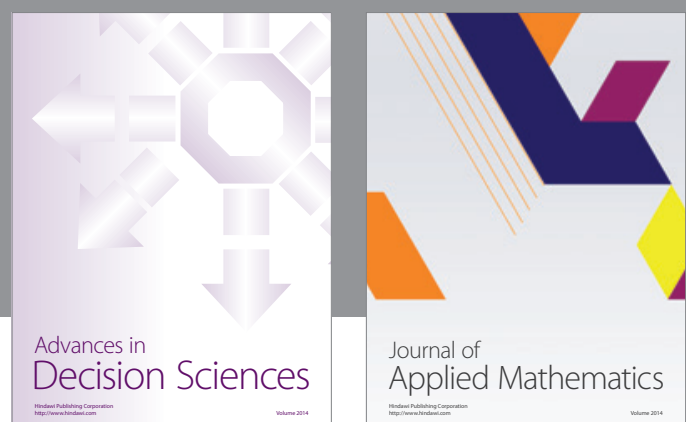

Journal of

Applied Mathematics
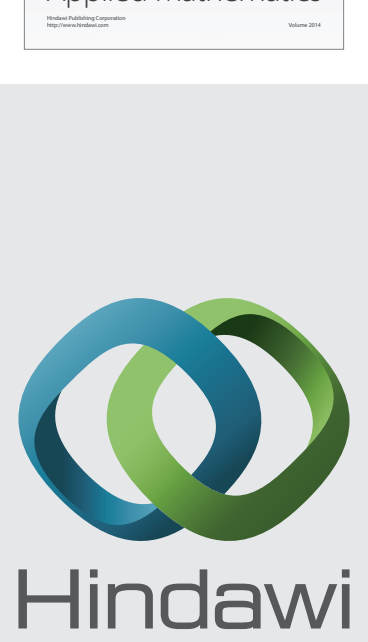

Submit your manuscripts at http://www.hindawi.com
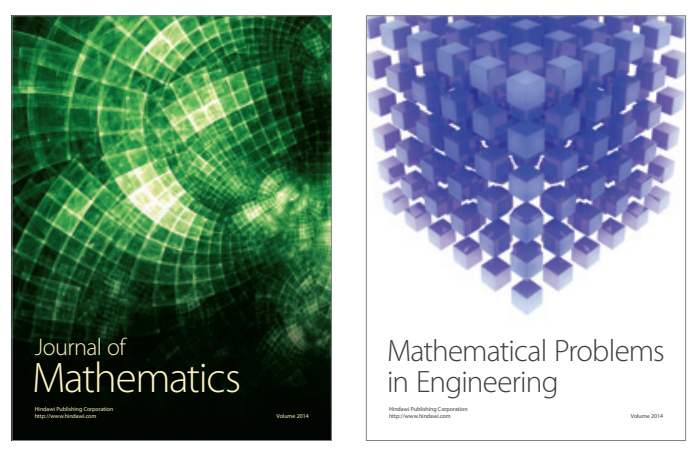

Mathematical Problems in Engineering
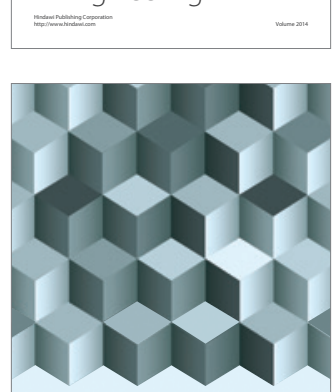

Journal of

Function Spaces
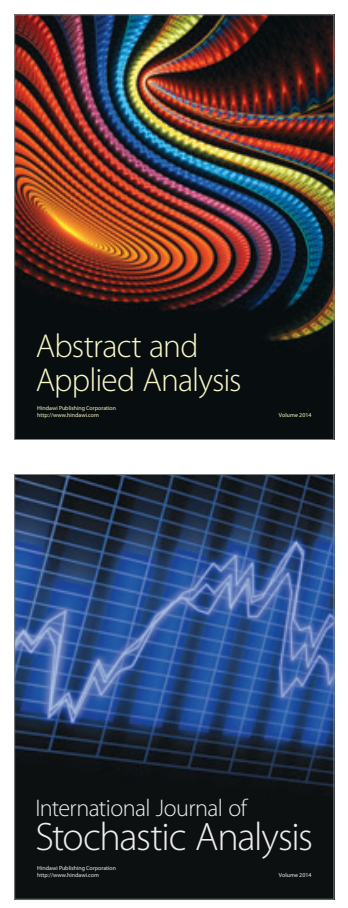

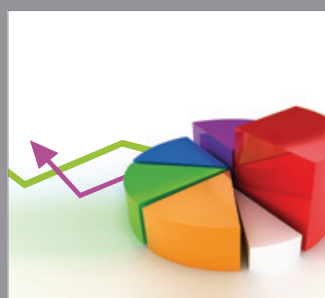

ournal of

Probability and Statistics

Promensencen
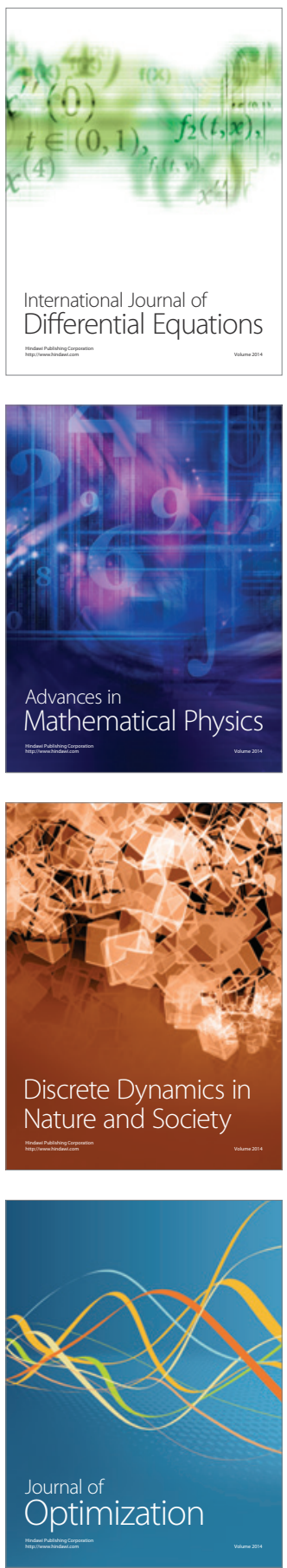